\title{
Characterization of Core Shell Cu-Au Nanoparticles by Microscopy Techniques.
}

\author{
Hector A. Calderon*, Yuping Bao**, Tianlong Wen*** and Kannan M. Krishnan*** \\ * Departamento de Física, ESFM-IPN, Ed. 9 UPALM Zacatenco, Mexico 07738. \\ ** Department of Chemical and Biological Engineering, University of Alabama, Tuscaloosa, \\ AL35487 \\ *** Department of Materials Science, University of Washington, Seattle, WA 98195-2120
}

Nanoparticles have attracted considerable attention due to their unique properties. Below a certain size, around $100 \mathrm{~nm}$, nanoparticles show different chemical and physical properties from bulk materials and atoms. Such properties depend on size, shape, structure and morphology and thus it is not only necessary to develop robust methods to synthesize nanoparticles but also characterize them. Normally, a monodispersed size distribution or a given particle shape are necessary for application of nanoparticles in diverse fields such as biology or energy production. In the present case, immiscible binary Co-Au core shell nanoparticles, with dual magnetic optical functionality and potential in biological application are investigated. Their sizes and shapes as a function of diverse parameters of synthesis is important as well as basic nucleation and growth processes of both core and shell (CS).

Co-Au nanoparticles are synthesized by colloidal techniques, using a hot injection method [1-3]. Figure 1 shows an example of nanoparticles as observed by STEM and TEM techniques. The atomic number contrast is clearly seen and the CS configuration becomes apparent (Fig. 1a). There is a size distribution in the resulting particles but the CS is developed for most particles. In some specific cases, larger particles are seen as single phase but this is most likely due to the thickness (particle size) dependence of the imaging technique. Au is heavier than Co giving rise to the contrast in the image but as particle size increases, the volume/thickness of the core dominates. Figure 1b shows an example of these nanoparticles as seen by high resolution in transmission electron microscopy. The different crystalline domains can be seen in this nanoparticle very clearly, due to its relatively small size. Larger particles show a contrast in TEM slightly more complicated to interpret. Figure 2 shows a typical case for a larger particle size. The core and the shell show relatively smaller differences but the use of Fourier transforms indicates the clearly different structures of shell and core showing that such differences are significant. As for the chemical composition of CS, single particles have been investigated by EELS and EDS techniques. However the size of the nanoparticles limits the statistical significance of the measurements. Thus EDS and EELS have been performed over arrays of particles as is shown in Figure 3 with an EDS spectrum and several scanned maps for different elements. Cobalt and gold signals are clearly separated, which proves that the cobalt in the core is distinct from the gold of the shell in the particles. These results show that the CS structure is developed with a polycrystalline shell that is promoted by heterogeneous nucleation of several nucleation sites of the shell during the synthesis process.

References

[1] Y. Bao, H. Calderon and Kannan M. Krishnan, Jour. Phys. Chem. C111, 1941-1944 (2007)

[2] V. F. Puntes and K. M. Krishnan, IEEE Trans. Mag. 37, 2210-2213 (2001)

[3] V. F. Puntes, Kannan M. Krishnan and P.A. Alivisatos, Science, 291, 2115-2117 (2001). 
[4] This research was supported by CONACYT (Proyecto 51833), IPN (COFAA-SIP) and US NSF/DMR \#0501421.
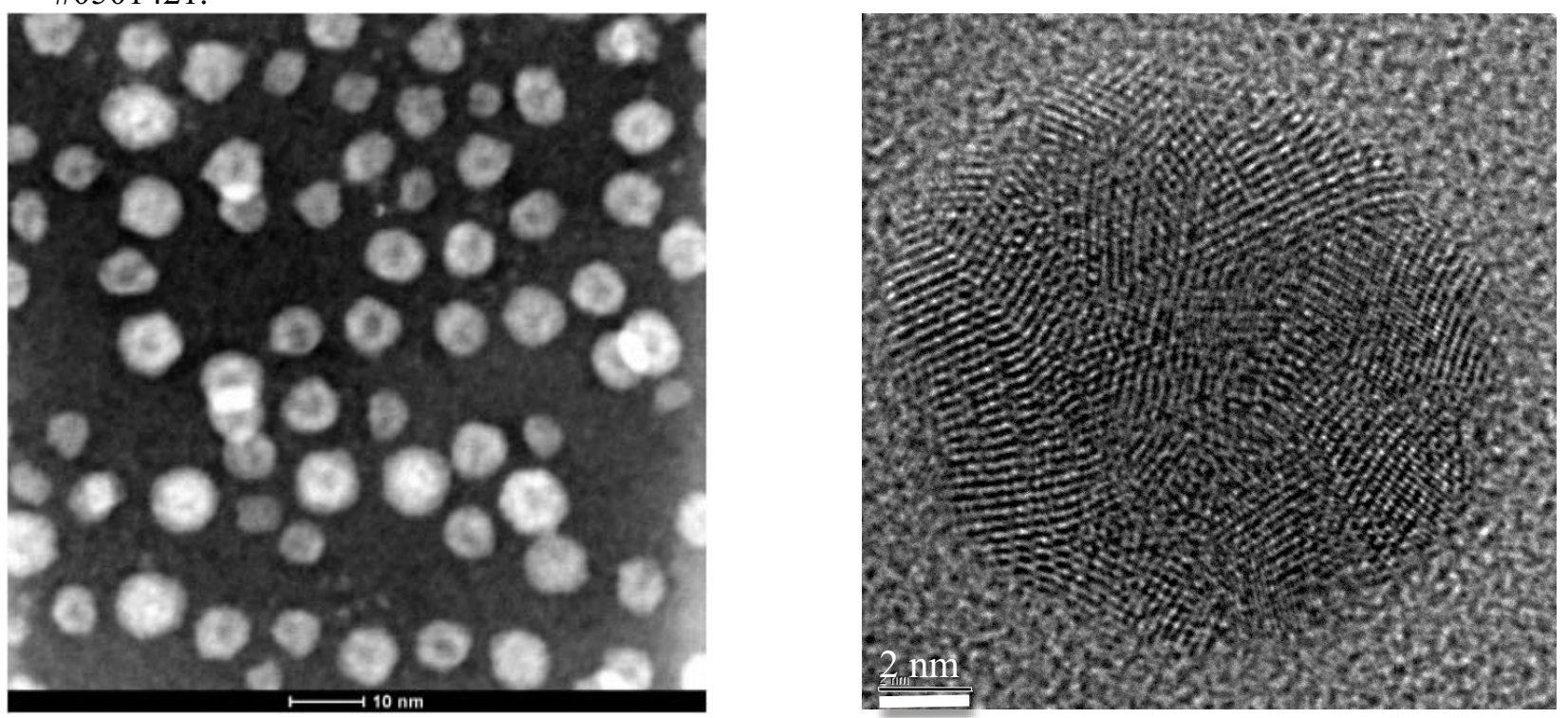

Figure 1. Core shell Co-Au particles as seen by (a) STEM and (b) High resolution TEM. The polycrystalline nature of the sheel is clarly seen.

Fig. 2. Core Shell particle of larger size. The core shell structure is easily identified by the Fourier transform taken at different locations.
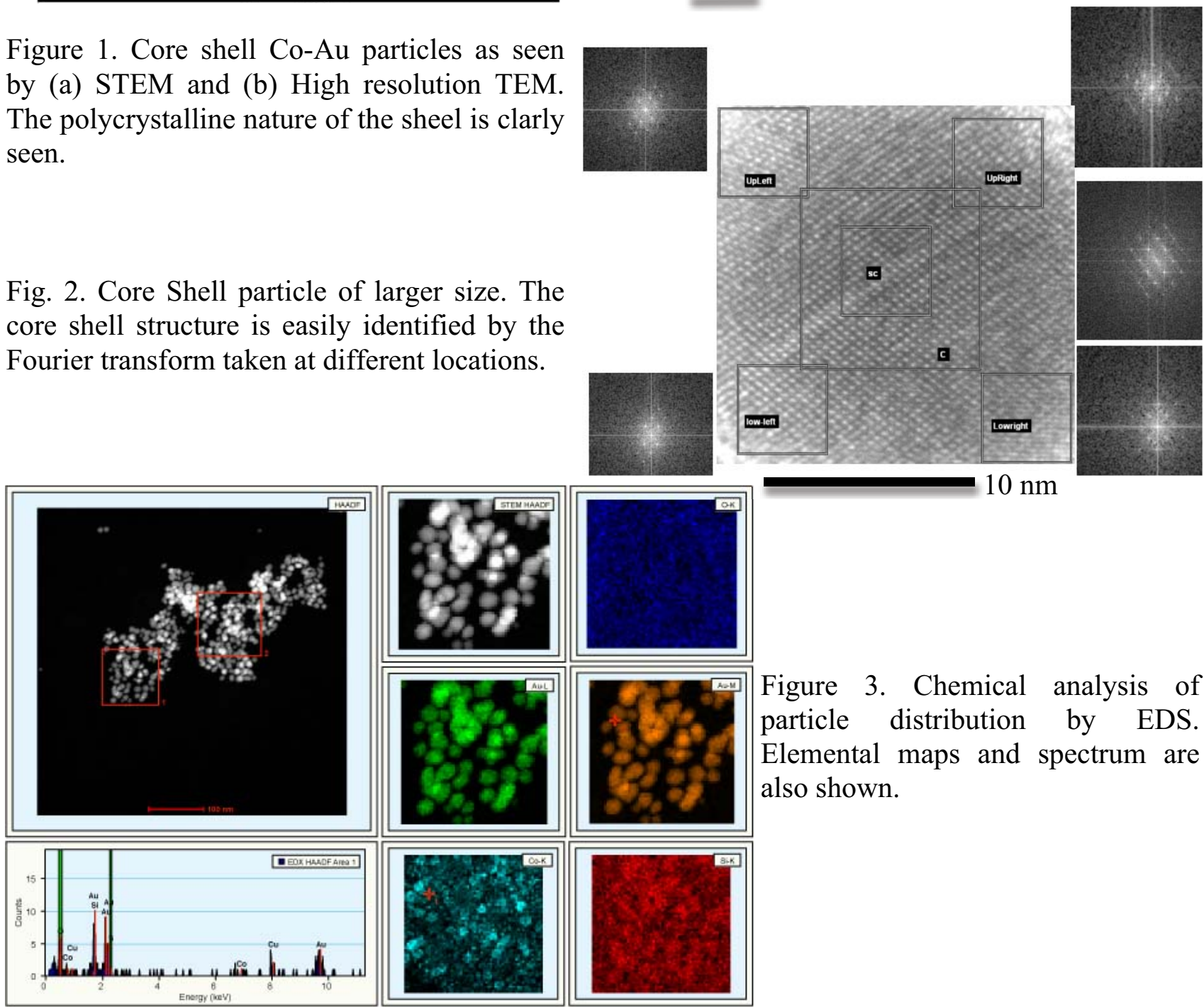

Figure 3. Chemical analysis of particle distribution by EDS. Elemental maps and spectrum are also shown. 